\title{
Special focus on artificial intelligence for optical communications*
}

Artificial intelligence (AI) and its related techniques bring new vitality and provide new approaches to optical communications, and enable new system design and thus new industries. Therefore, research attention and dedication have been attracted continuously from both academia and industry. In particular, combining AI and existing techniques in optical communication systems and networks, such as optical signal processing, has shown tremendous potential to offer promising solutions to performance improvement and network optimization in the areas of broadband optical access, high speed optical transmission, flexible optical switching, as well as intelligent optical networking. However, applying AI techniques to optical communications is still in its infancy, leading to a number of challenges that need to be urgently addressed, in research, development and application.

To report the latest research progress in this area, we have organized a special focus feature on artificial intelligence for optical communications in SCIENCE CHINA Information Sciences. After rigorous peer review process, the special focus accepts five scientific articles, with themes involving optical network architecture, optical signal processing, optical parameter optimization, and optical transmission algorithm.

"Artificial intelligence-driven autonomous optical networks: 3S architecture and key technologies" proposes a "3S" autonomous optical networks (AON) which consists of "self-aware" network status, "self-adaptive" network control, and "self-managed" network operations. Various AI techniques are incorporated into AON from the device, link and network aspects, to promote autonomous management.

"An overview of ML-based applications for next generation optical networks" reviews machine learning (ML) technologies for optical networks. The authors focus on a number of challenging issues, including the low-margin network design, power optimization, routing and wavelength assignment, as well as failure management.

"AI based on frequency slicing deep neural network for underwater visible light communication" presents a low-complexity frequency slicing deep neural network (FSDNN) for wide-band signal postequalization in a $1.2 \mathrm{~m}$ underwater visible light communication system.

In "Intent defined optical network with artificial intelligence-based automated operation and maintenance", an optical network architecture towards AI-based optical network automated operation and maintenance is designed, where a self-adapted generation and optimization (SAGO) policy is introduced.

In "Overfitting effect of artificial neural network based nonlinear equalizer: from mathematical origin to transmission evolution", the overfitting effect of AI-based nonlinear equalizer in training and testing

\footnotetext{
*Citation Ji Y F, Lu C, Zibar D, et al. Special focus on artificial intelligence for optical communications. Sci China Inf Sci, 2020, 63(6): 160300, https://doi.org/10.1007/s11432-020-2898-2
} 
is studied in the context of the optical fiber communication system.

Finally, we would like to express our sincere appreciation to all the authors for submitting their latest research outcomes. Besides, we express our deepest gratitude to all the anonymous reviewers for delivering high-quality and timely review comments. Last but not least, we thank SCIENCE CHINA Information Sciences Editorial Office for the scrupulous service and supports during the whole process of this special focus.

Guest Editors:

Yuefeng JI

Beijing University of Posts and Telecommunications, China

Chao LU

The Hong Kong Polytechnic University, China

Darko ZIBAR

Technical University of Denmark, Denmark

Huanlai XING

Southwest Jiaotong University, China 